\title{
" El Régimen de Tipo de Cambio y la Composición de la Deuda Corporativa: La Experiencia Mexicana "
}

\author{
Lorenza Martínez \\ lmartin@banxico.org.mx \\ Alejandro Werner ${ }^{1 / 2 /}$ \\ awerner@banxico.org.mx
}

Febrero 2002

Documento de Investigación No. 2002-02

Dirección General de Investigación Económica

BANCO DE MÉXICO

\footnotetext{
${ }^{1 /}$ Documento preparado para el Seminario Interamericano de Economía de Verano organizado por el NBER, Julio 20-21, 2001.

${ }^{2 /}$ Los autores laboran en la Dirección de Investigación Económica del Banco de México. Agradecemos a Judith Frías, Roberto Romero, Eduardo Espinosa y Daniel Acevedo por su colaboración en la investigación y a Marc Aguiar y demás participantes en el Seminario Interamericano de Economía de Verano organizado por el NBER por sus comentarios. Los autores son responsables por las opiniones expresadas en el documento y no representan las del Banco de México. Mayores informes: Tel. (525) 237.25.73, Dirección: Av. 5 de Mayo \#18, piso 4, Col. Centro, D.F. 06059, México.
} 


\title{
" El Régimen de Tipo de Cambio y la Composición de la Deuda Corporativa: La Experiencia Mexicana "
}

Lorenza Martínez

Alejandro Werner

\author{
Diciembre 2001 \\ Documento de Investigación No. 2001 - 02 \\ Dirección General de Investigación Económica \\ BANCO DE MÉXICO
}

\begin{abstract}
$\underline{\text { Resumen }}$
En este artículo se analiza el efecto que el cambio en el régimen cambiario de fijo a flexible, ocurrido en México en diciembre de 1994, tuvo sobre la composición de la deuda corporativa. En particular, se evalúa la tesis de si un régimen de tipo de cambio fijo induce un sobreendeudamiento en moneda extranjera, debido a la garantía implícita otorgada por el gobierno contra una devaluación del tipo de cambio. Esto es, bajo un régimen de tipo de cambio predeterminado las empresas no internalizan completamente su riesgo cambiario y tenderán a ser más propensas a incurrir en desequilibrios cambiarios en sus balances. Con este fin en el artículo se estudian los principales determinantes del endeudamiento en moneda extranjera en que incurrieron las empresas listadas en la Bolsa Mexicana de Valores durante el período de 1992 a 2000. La finalidad es probar si en efecto los desequilibrios cambiarios en los balances financieros disminuyeron a raíz de la adopción del régimen de tipo de cambio flexible. Los resultados respaldan la tesis de que el régimen de tipo de cambio flexible reduce la exposición cambiaria de las empresas.
\end{abstract}




\section{Introducción}

En este artículo se analiza el efecto que el cambio en el régimen cambiario de fijo a flexible, ocurrido en México en diciembre de 1994, tuvo sobre la composición de la deuda corporativa. En particular, se evalúa la tesis de si un régimen de tipo de cambio fijo induce un sobreendeudamiento en moneda extranjera, debido a la garantía implícita otorgada por el gobierno contra una devaluación del tipo de cambio. Esto es, bajo un régimen de tipo de cambio predeterminado las empresas no internalizan completamente su riesgo cambiario y tenderán a ser más propensas a incurrir en desequilibrios cambiarios en sus balances. Con este fin en el artículo se estudian los principales determinantes del endeudamiento en moneda extranjera en que incurrieron las empresas listadas en la Bolsa Mexicana de Valores durante el período de 1992 a 2000. La finalidad es probar si en efecto los desequilibrios cambiarios en los balances financieros disminuyeron a raíz de la adopción del régimen de tipo de cambio flexible.

Un punto central en la polémica actual acerca de la reforma de la arquitectura financiera internacional es el impacto del régimen cambiario sobre la vulnerabilidad financiera. Uno de los principales canales a través de los cuales el régimen de tipo de cambio puede afectar la vulnerabilidad de una economía es a través de su repercusión en el endeudamiento en moneda extranjera de los agentes económicos. Sin embargo, no hay un consenso claro entre los economistas respecto a esta discusión.

Por un lado, varios autores han argumentado que un esquema de tipo de cambio fijo está asociado con el otorgamiento de garantías implícitas. La razón, afirman, es que para mantener un régimen de este tipo la autoridad monetaria siempre afirmará que las perspectivas de un cambio en la paridad son nulas. Mediante esa negación constante de la posibilidad de un cambio en la paridad, las autoridades estarán asumiendo implícitamente

parte del costo que el sector privado incurriría en caso de una devaluación. En estas circunstancias, los agentes del sector privado tendrán menos incentivos para cubrir su apalancamiento en moneda extranjera. 
Varios autores han esgrimido recientemente este tipo de argumentos. Por ejemplo, Fisher (2001) indica claramente los incentivos que llevan a un sobreendeudamiento en moneda extranjera en un régimen cambiario predeterminado:

"La creencia de que el tipo de cambio no cambiará elimina la necesidad de cobertura, y reduce la percepción de riesgo de la deuda denominada en moneda extranjera ".

Siguiendo la misma línea, Mishkin (1996) destaca las ventajas de un régimen de tipo de cambio flexible:

"Sin duda, la fluctuación diaria del tipo de cambio en un régimen de tipo de cambio flexible tiene la ventaja de poner en claro a las empresas, bancos y gobiernos que existe un riesgo sustancial implícito en la emisión de deuda en moneda extranjera ".

Por otro lado, sin descartar totalmente el argumento de las garantías implícitas, otros autores aseguran que en algunos mercados emergentes existe una tendencia natural hacia la dolarización de los pasivos, efecto más arraigado al sistema de lo que puede explicar un régimen de tipo de cambio predeterminado. A este argumento se le ha llamado la hipótesis del pecado original (véase Eichengreen y Hausmann (2000)). Dichos autores definen a la situación del pecado original de la siguiente manera:

"Es la situación en la cual la moneda local no puede utilizarse para pedir prestado en el exterior o para pedir prestado a largo plazo, incluso internamente. En esta situación de mercados incompletos, la fragilidad financiera es inevitable porque todas las inversiones internas tendrán un desequilibrio cambiario (proyectos que generan pesos serán financiadas con dólares) o discordancias en el plazo (proyectos de largo plazo serán financiados con préstamos de corto plazo).

Ciertamente, los desequilibrios descritos existen no porque los bancos y las empresas carezcan de la prudencia para cubrir sus deudas. El problema, en cambio, es que un país cuyas obligaciones externas están necesariamente denominadas en moneda extranjera es, por definición, incapaz de cubrirse. Suponer que habrá alguien del otro lado del mercado que realizará las coberturas en moneda extranjera es equivalente a suponer que el país puede pedir prestado en el exterior en su propia moneda. De igual manera, el problema no 
es simplemente que las empresas carezcan de la previsión necesaria para igualar el plazo de sus activos y pasivos; la realidad más bien es que encuentran imposible lograrlo. La raíz de la fragilidad financiera está en lo incompleto de los mercados financieros. En consecuencia, tanto un régimen de tipo de cambio fijo como uno flexible son problemáticos."

Otros autores (por ejemplo Calvo y Reinhart (2000 (a) y (b)) han puesto énfasis en argumentos similares diciendo que el problema de la falta de cobertura de las obligaciones en moneda extranjera tiene raíces más profundas que la elección de un régimen cambiario. Por tanto, según estos autores, el movimiento hacia regímenes flexibles que está teniendo lugar en los mercados emergentes no aliviará este problema.

Aunque la discusión ha sido intensa, no hay estudios empíricos enfocados a esta controversia. Y debemos decir que en última instancia ésta es principalmente una pregunta empírica. La experiencia mexicana reciente representa un buen caso de estudio para abordar dicha pregunta. México fue el primer país en sufrir una "Crisis del Siglo XXI" (que comenzó con la devaluación del peso el 19 de diciembre de 1994) y en adoptar un régimen de tipo de cambio flexible después de ésta. Además, la disponibilidad de datos permite estudiar los determinantes de la deuda corporativa en moneda extranjera.

En este trabajo se toman las dos hipótesis mencionadas para argumentar que se complementan recíprocamente. El hecho de que un país no pueda emitir deuda en su propia moneda no significa que los acreedores no deban considerar los riesgos cambiarios que asumen al prestarle a empresas mexicanas. Y aunque no exista un mercado de coberturas lo suficientemente desarrollado, las empresas tienen exposiciones cambiarias diversas dependiendo de si venden en mercados locales o externos. Debido a esto, los acreedores pueden ajustar el nivel de riesgo cambiario seleccionando diferentes tipos de empresas. Por tanto, es posible que en una economía que sufre de pecado original, un tipo de cambio fijo lleve consigo un seguro cambiario implícito y consecuentemente ello incentive a esa economía al endeudamiento en moneda extranjera.

Para formalizar estos argumentos, en la sección II se amplía el modelo desarrollado por Holmstrom y Tirole (1997), para tomar en cuenta la posibilidad de desequilibrios 
cambiarios. En nuestra versión del modelo las empresas pueden pedir prestado en pesos a los bancos locales, los cuales supervisan su comportamiento, y en dólares en los mercados internacionales de capital donde el costo del capital es menor. Esto es, se modela la hipótesis de Eichengreen y Hausmann para derivar implicaciones que puedan ser probadas empíricamente. De este modelo se desprenden resultados claros sobre los determinantes de la proporción de la deuda en dólares sobre la deuda total. Las empresas con una mayor probabilidad de éxito en sus proyectos de inversión, con un problema de información menor y más orientadas a la exportación, tendrán una mayor proporción de deuda denominada en dólares y un mayor apalancamiento. También, debido a que con un régimen de tipo de cambio fijo habrá una garantía implícita, bajo este esquema la orientación exportadora de la empresa será relativamente menos importante en la determinación de la deuda denominada en moneda extranjera. Así, en este marco, aumentará la participación de esta última respecto a la deuda total y se producirá un mayor apalancamiento.

En la sección III se describe la evolución de la proporción de la deuda denominada en dólares sobre la deuda total para las empresas mexicanas de 1992 a 2000. A continuación con base a las implicaciones del modelo probamos empíricamente la validez de la hipótesis propuesta. Una conclusión es que aunque la mediana de la proporción de la deuda en dólares sobre la total aumentó de 32\% en 1994 a $42 \%$ en 2000, la exposición ${ }^{3 /}$ al riesgo cambiario disminuyó durante dicho período. La mediana del cociente de los ingresos por exportaciones y la deuda en dólares aumentó de 2\% en 1994 a 7.4\% en 2000.

Al estimar la ecuación derivada del modelo, correspondiente a los determinantes de la razón de la deuda en dólares y la total, se encontró que por un lado mientras prevaleció el régimen de tipo de cambio predeterminado nuestra variable endógena estuvo explicada principalmente por el tamaño de las empresas deudoras. Por otro lado, en la etapa del régimen de libre flotación, las exportaciones se convirtieron en la única variable significativa determinante de la importancia del endeudamiento en dólares. Estos resultados son robustos incluso cuando se controla por el crecimiento de las ventas.

\footnotetext{
${ }^{3 /}$ A lo largo del documento se entiende como exposición al riesgo cambiario de las empresas al desbalance entre los ingresos y egresos denominados en dólares. En particular se utiliza la razón de exportaciones con respecto a la deuda denominada en dólares para medir esta "exposición" o desbalance.
} 
Finalmente, en la sección IV se presentan las conclusiones principales del trabajo.

\section{El Modelo}

El modelo aquí presentado es una ampliación del modelo desarrollado por Holmstrom y Tirole (1997) para analizar las alternativas de financiamiento para una empresa que tiene que elegir un nivel de inversión (I) y enfrenta un monto limitado de recursos internos (A). Cuando el nivel deseado de la inversión excede al de los activos de la empresa, ésta tendrá que financiar el remanente ya sea a través de la banca local que presta en pesos (con vencimiento a corto plazo $)^{4 /}$ o en mercados extranjeros que prestan en dólares.

El contrato deberá especificar cuánto invertirá cada parte y los pagos a cada uno de ellos en función del resultado del proyecto. Un contrato óptimo tendrá la siguiente estructura:

(i) La empresa invierte todo su capital A.

(ii) Todos reciben un pago de 0 en caso de que el proyecto falle.

(iii) Cuando el proyecto es exitoso, la empresa recibe un pago $R_{f}>0$, el banco local $R_{b}>0$ y los acreedores extranjeros $E_{t+1} R_{u}>0$ (donde $R_{u}$ es el rendimiento en dólares y $E_{t+1}$ el tipo de cambio del período siguiente).

Las empresas enfrentan un problema de riesgo moral, dado que en ausencia de una supervisión apropiada, ellas tienden deliberadamente a reducir su probabilidad de éxito y conseguir a cambio un beneficio (B). Cuando se opta por esta línea de acción, la probabilidad de éxito cae de $P_{H}$ a $P_{L}$ (llamamos $\left.P=P_{H}-P_{L}>0\right)$. Así, el proyecto tiene un rendimiento por unidad invertida igual a:

$$
R=S_{d} E_{t+1}+S_{P}-W_{P}
$$

Donde: $\quad S_{d}$ : son las ventas en dólares

\footnotetext{
4/ La decisión de prestar a corto plazo, aunque es irrelevante para el modelo presentado, podría explicarse como una parte instrumental del papel de supervisión que realizan los bancos locales.
} 


$$
\begin{aligned}
& S_{P}: \text { son las ventas en pesos } \\
& W_{P}: \text { costo (en pesos) } \\
& E_{t+1}: \text { el tipo de cambio en } \mathrm{t}+1
\end{aligned}
$$

El papel de los bancos locales en este modelo es el de supervisar a las empresas y abatir los problemas de riesgo moral. Aunque los bancos realicen esta tarea no son capaces de eliminar por completo el problema de riesgo moral y sólo pueden reducir el beneficio privado de $B$ a $b$. Para desempeñar su función de seguimiento, los bancos pagarán una cantidad no observable $c$ por cada unidad de inversión. A fin de recuperar este costo deberán prestar una cantidad suficiente de fondos al proyecto a una tasa por encima de la tasa de mercado. Debido al hecho de que las fuentes de recursos están principalmente en pesos, se supone que todos los préstamos bancarios están en pesos y exigen una tasa de rendimiento $r$. Finalmente, se supone que los acreedores extranjeros son uniformes y demandan una tasa de rendimiento en moneda extranjera igual a $r^{*}$.

Bajo este esquema, una empresa racional elegirá $I, R_{b}, R_{u}, I_{b}, I_{u}$ dado $r^{*}, r$ y $A$, para:

$$
\text { Maximizar: } U(A)=P_{H}\left[S_{d} E_{t+1}+S_{P}-W_{P}\right] I-P_{H} R_{b}-P_{H} R_{u} E_{t+1}
$$

Sujeto a:

(i) $\quad A+I_{b}+I_{u} E_{t}=I$

(ii) $P_{H} R_{b} \geq r I_{b}$

(iii) $P_{H} R_{u} \geq r^{*} I_{u}$

(iv) $\quad P R_{b} \geq c I$

(v) $\quad P R_{f} \geq b I$

(vi) $\quad R_{f}+R_{b}+E_{t+l} R_{u} \leq R I$

\footnotetext{
${ }^{5 /}$ Como la vigilancia es costosa y los bancos podrían en principio invertir en el extranjero a una tasa $\mathrm{r}^{*}$, la tasa de interés interna $\mathrm{r}$ ajustada por los costos de vigilancia y por las expectativas de depreciación debe ser
} 
La primera restricción, o restricción presupuestal, indica que la inversión total tiene que ser financiada mediante una de las tres fuentes mencionadas: capital de la empresa (A), crédito de los bancos nacionales $\left(\mathrm{I}_{b}\right)$ y préstamos en moneda extranjera $\left(\mathrm{I}_{\mathrm{u}}\right)$. Las restricciones (ii) y (iii) garantizan que la tasa esperada de rendimiento para los bancos y para los acreedores extranjeros debe ser por lo menos de $r$ (en pesos) y de $r^{*}$ (en dólares), respectivamente. Las restricciones (iv) y (v) son las de compatibilidad de incentivos, para que las empresas consideren los proyectos con alta probabilidad de éxito y para que los bancos realicen la tarea de supervisión. Finalmente, la ecuación (vi) indica que el rendimiento total del proyecto tiene que ser dividido entre la empresa, el banco y los acreedores extranjeros.

Debido a la naturaleza de rendimientos constantes de la función de beneficio de la empresa, es obvio que en equilibrio todas las restricciones se cumplirán con igualdad. De (iv) y (v) se obtiene el rendimiento respectivo que la empresa y los bancos internos requieren como garantía para que sean los proyectos con alta probabilidad de éxito los que se consideren y para que los bancos lleven a cabo la supervisión esperada.

$$
R_{b}=\frac{c I}{\Delta P}
$$$$
R_{f}=\frac{b I}{\Delta P}
$$

Lo anterior define lo que Holmstrom y Tirole llaman ingreso "comprometible" esperado. Éste es el ingreso esperado que puede ser ofrecido a los inversionistas extranjeros a la vez de que se mantienen los incentivos de las empresas y de la banca local. Dicho concepto denominado en pesos será igual a:

$$
P_{H}\left[S_{d} E_{t+1}+S_{p}-W_{p}-\frac{(c+b)}{\Delta P}\right] I
$$

Este rendimiento traducido a dólares debe ser suficiente para satisfacer la restricción (iv). Por tanto, de la ecuación (4) es claro que el ingreso "comprometible" esperado en dólares es decreciente en el tipo de cambio del siguiente período. Y que la sensibilidad a modificaciones del tipo de cambio esperado está relacionada con la importancia del 
superávit en pesos $\left(S_{p}-W_{p}-\frac{(c+b)}{\ddot{A} P}\right)$ del rendimiento total de las empresas. Mientras $S_{d}$ sea mayor, el ingreso "comprometible" esperado será menos sensible al movimiento esperado del tipo de cambio.

$$
P_{H}\left[S_{d}+\frac{S_{p}-W_{p}-\frac{(c+b)}{\ddot{A} P}}{E_{t+1}}\right] I=r^{*} I_{u}
$$

Como lo muestra la ecuación (5), el nivel del apalancamiento en moneda extranjera estará negativamente relacionado con la depreciación esperada del tipo de cambio (asumiendo que la empresa tiene un superávit en pesos). Esta sensibilidad será menor mientras más orientada esté la empresa hacia la exportación. Así, el nivel de inversión por los acreedores extranjeros estará determinado por:

$$
I_{u}=\frac{P_{H}}{r^{*}}\left[S_{d}+\frac{\left(S_{p}-W_{p}\right)}{E_{t+1}}-\frac{(c+b)}{\Delta P E_{t+1}}\right] I
$$

Las expresiones (1) y (ii) también determinan el nivel necesario de inversión por parte de los bancos locales $\left(\mathrm{I}_{\mathrm{b}}\right)$ para que obtengan un rendimiento $\mathrm{r}$ e incentivarlos a supervisar:

$$
I_{b}=\frac{P_{H}}{r} \frac{c I}{\Delta P}
$$

De (5) y (6) se deriva la proporción de la deuda denominada en dólares sobre la deuda total:

$$
\frac{I_{u} E_{t}}{I_{u} E_{t}+I_{b}}=\frac{\frac{P_{H}}{r^{*}}\left[S_{d} E_{t}+\left(S_{p}-W_{p}-\frac{(c+b)}{\Delta P}\right) \frac{E_{t}}{E_{t+1}}\right]}{\frac{P_{H}}{r^{*}}\left[S_{d} E_{t}+\left(S_{p}-W_{p}-\frac{c+b}{\Delta P}\right) \frac{E_{t}}{E_{t+1}}\right]+\frac{P_{H}}{r} \frac{c}{\Delta P}}
$$

La ecuación (7) muestra que dado que la deuda en dólares es la fuente marginal de financiamiento, su proporción respecto a la deuda total aumentará a medida que los costos de supervisión (c) sean menores, los beneficios privados (b) caigan, la probabilidad de éxito

mantendrá (para más detalles revisar Holmstrom y Tirole (1997). 
$\left(\mathrm{P}_{\mathrm{H}}\right)$ aumente y que las tasas de interés, locales y extranjeras, bajen. Reordenando la ecuación (7) obtenemos la ecuación (8), que podemos estimar empíricamente:

$$
\frac{I_{u} E_{t}}{I_{u} E_{t}+I_{b}}=\underbrace{\frac{P_{H}}{r^{*}}}_{\alpha} \frac{\text { Exp }}{\text { Deuda Total }}+\underbrace{\frac{P_{H}}{r^{*}} \frac{E_{t}}{E_{t+1}}}_{\beta}\left[\frac{\text { Vtas Domest Netas }}{\text { Deuda Total }}-\frac{(c+b) / \Delta P}{\text { Deuda Total }}\right]
$$

De la ecuación (8) queda claro que la participación de la deuda en dólares se relacionará negativamente con la tasa de depreciación esperada, mientras la empresa tenga un superávit en pesos. Esto es así porque cuando el ingreso "comprometible" esperado en dólares disminuye, el financiamiento disponible en dólares cae. Este efecto será menor para las empresas más orientadas a la exportación dado que su ingreso "comprometible" en dólares será menos sensible a las fluctuaciones cambiarias. Además, en la medida en que los agentes económicos perciban que hay un nivel de tipo de cambio garantizado por el gobierno $\left(E_{t+1}=E_{t}\right)$, los acreedores extranjeros no tomarán en cuenta la vulnerabilidad al tipo de cambio por parte de la empresa como un determinante de la proporción de la deuda en dólares sobre la total.

En la sección empírica se estima la ecuación (8) y se evalúa la hipótesis de que durante el régimen de tipo de cambio predeterminado, la devaluación esperada era muy pequeña o nula y por ende $\alpha=\beta$. En contraste, durante el régimen de flotación, se contempla un riesgo de devaluación de manera que $\alpha>\beta$. Bajo la hipótesis alternativa no existe diferencia alguna entre los coeficientes de ambos períodos, de manera que durante ambos períodos se subestima el riesgo cambiario $(\alpha=\beta)$. Asimismo, puede ser que $\alpha>\beta$, de modo que bajo los dos regímenes se considere con la misma importancia al riesgo cambiario.

Insertando (5) y (6) en (i) obtenemos el nivel de equilibrio de la inversión:

$$
\begin{gathered}
A+\left[\frac{P_{H}}{r^{*}}\left\{S_{d} E_{t}+\left(S_{p}-W_{p}-\frac{(c+b)}{\Delta P}\right) \frac{\mathrm{E}_{\mathrm{t}}}{E_{t+1}}\right\}+\frac{\mathrm{P}_{\mathrm{H}}}{\mathrm{r}} \frac{c}{\Delta P}\right] I=I \\
I=\frac{A}{1-\frac{P_{H}}{r^{*}}\left\{S_{d} E_{t}+\left(S_{p}-W_{p}-\frac{(c+b)}{\Delta P}\right) \frac{E_{t}}{E_{t+1}}\right\}-\frac{P_{H}}{r} \frac{c}{\Delta P}}
\end{gathered}
$$


De ello se sigue que los principales determinantes de la inversión total son los mismos que rigen para la proporción de la deuda en dólares sobre la deuda total. Este resultado es obvio debido al hecho de que la deuda en dólares es la fuente marginal de financiamiento. De la ecuación (9) podemos derivar una expresión para la relación entre deuda y capital:

$$
\frac{\text { Deuda Total }}{\text { Capital }}=\frac{\frac{P_{H}}{r^{*}}\left\{S_{d} E_{t}+\left(S_{p}-W_{p}-\frac{(c+b)}{\Delta P}\right) \frac{E_{t}}{E_{t+1}}\right\}+\frac{P_{H}}{r} \frac{c}{\Delta P}}{1-\frac{P_{H}}{r^{*}}\left\{S_{d} E_{t}+\left(S_{p}-W_{p}-\frac{(c+b)}{\Delta P}\right) \frac{E_{t}}{E_{t+1}}\right\}-\frac{P_{H}}{r} \frac{c}{\Delta P}}
$$

y a partir de (10) la ecuación (11) a estimar está dada por:

$$
\frac{\text { Deuda Total }}{\text { Capital }}=\underbrace{\frac{P_{H}}{r^{*}}}_{\alpha} \frac{\text { Exp }}{\text { Capital }}+\underbrace{\frac{P_{H}}{r^{*}} \frac{E_{t}}{E_{t+1}}}_{\beta}\left[\frac{\text { Vtas Domest Netas }}{\text { Capital }}-\frac{(c+b) / \Delta P}{\text { Capital }}\right]+\frac{P_{H}}{r} \frac{c}{\Delta P}
$$

de donde podría realizarse una prueba alternativa de nuestra hipótesis.

\section{Análisis Empírico}

En esta sección se presentan la base de datos utilizada para el trabajo, algunas estadísticas básicas de las variables empleadas, los hechos estilizados más importantes y los resultados econométricos. Los datos se obtienen de la Bolsa Mexicana de Valores e incluyen no solamente a las empresas con acciones listadas sino también a las que han emitido deuda. El período cubierto se extiende de 1992 a 2000. El número total de empresas es 376, pero muy pocas (81) se encuentran presentes durante todo el período.

Se construyó una matriz de transición para analizar la distribución de las empresas de acuerdo con las dos principales variables de interés: exportaciones y endeudamiento en dólares. El Cuadro 1, que muestra la matriz, también ayuda a constatar cómo las características de las empresas en la muestra cambiaron de 1992 a 2000. Se eligió a 1992 como el año inicial para no considerar el período previo a la liberalización financiera y así evitar los cambios en la estructura de la deuda propiciados por este proceso. La matriz incluye cinco categorías por año, resultando en un total de 25 grupos que describen el estatus de cada empresa en el año inicial y final. Las filas corresponden al número de 
empresas en cada categoría durante 1992, mientras que las columnas se refieren a las mismas categorías para 2000. Como de costumbre, los términos en la diagonal corresponden a aquellas observaciones que no cambiaron de estatus entre los dos años y lo contrario se aplica para los términos fuera de la diagonal. La última fila y la última columna muestran el número total de empresas en cada una de las cinco categorías en 1992 y 2000 respectivamente.

En el Cuadro 1 se muestra que el número total de empresas presentes ya sea en 1992 o en 2000 era de 318. De éstas 58 entraron después de 1992 y 179 salieron antes de o durante 2000. Solamente 81 empresas estuvieron presentes durante todo el período. La muestra, en ambos años, está dominada por las empresas con deuda denominada en dólares. Por ejemplo, 269 empresas, es decir 85\% del total, tenían deuda en dólares en alguno de los dos años, 215 (83\%) de las empresas la tenían en 1992 mientras que este número cambió a 124 (89\%) para 2000. Por tanto, aún en 1992 la mayoría de las empresas listadas en la Bolsa disponía de acceso al financiamiento en dólares y, sorpresivamente, la proporción de empresas apalancadas en dólares no aumentó significativamente. Con respecto a las tasas de supervivencia, 36 empresas sin deuda en dólares en 1992 salieron de la muestra, mientras que solamente 9 sobrevivieron hasta 2000. De estas últimas, únicamente 4 permanecieron sin pasivos en divisas. De la misma manera, solamente 9 (15\%) de las empresas que entraron después de 1992 no tenían deuda en dólares en 2000.

Con respecto a la categoría de exportador, vemos también que la mayoría de las empresas se ha encontrado en dicha situación, pero en este caso observamos un aumento importante en la participación de estas empresas de $58 \%$ a $68 \%$. Es también de notarse que la mayoría de las empresas exportadoras tiene deuda en dólares. Por ejemplo, 46 empresas o 57\% del número total de las presentes en ambos años corresponden a la categoría mencionada. Durante ambos años, solamente $2 \%$ de las empresas exportadoras no tenían obligaciones denominadas en dólares.

Es importante mencionar que además de las 318 empresas mencionadas, la muestra incluye otras 58 que entraron al mercado después de 1992 y salieron antes de 2000. Por tanto, el número total de empresas en la muestra es de 376. 


\begin{tabular}{|c|c|c|c|c|c|c|}
\hline \multirow[b]{2}{*}{$1992 \backslash 2000$} & \multicolumn{5}{|c|}{$\begin{array}{c}\text { Cuadro } 1 \\
\text { Matriz de Transición }\end{array}$} & \\
\hline & $\begin{array}{c}\text { No } \\
\text { existentes en } \\
1992\end{array}$ & $\begin{array}{l}\text { Deuda en } \\
\text { USD y } \\
\text { exportadoras }\end{array}$ & $\begin{array}{l}\text { Deuda en } \\
\text { USD y no } \\
\text { exportadoras }\end{array}$ & $\begin{array}{l}\text { Sin deuda en } \\
\text { USD y } \\
\text { exportadoras }\end{array}$ & $\begin{array}{c}\text { Sin deuda en } \\
\text { USD y no } \\
\text { exportadoras }\end{array}$ & \\
\hline $\begin{array}{l}\text { No existentes } \\
\text { en } 1992\end{array}$ & 0 & 36 & 13 & 1 & 8 & 58 \\
\hline $\begin{array}{l}\text { Deuda en } \\
\text { USD y } \\
\text { exportadoras }\end{array}$ & 97 & 46 & 4 & 1 & 0 & 148 \\
\hline $\begin{array}{l}\text { Deuda en } \\
\text { USD y no } \\
\text { exportadoras }\end{array}$ & 46 & 8 & 12 & 0 & 1 & 67 \\
\hline $\begin{array}{l}\text { Sin deuda en } \\
\text { USD y } \\
\text { exportadoras }\end{array}$ & 2 & 1 & 0 & 0 & 0 & 3 \\
\hline $\begin{array}{l}\text { Sin deuda en } \\
\text { USD y no } \\
\text { exportadoras }\end{array}$ & 34 & 2 & 2 & 0 & 4 & 42 \\
\hline & 179 & $\overline{93}$ & $\overline{31}$ & 2 & 13 & 318 \\
\hline
\end{tabular}

\section{III.1. Hechos estilizados}

A continuación se aborda el análisis del comportamiento de la composición de la deuda corporativa en términos de su denominación, ya sea en pesos o en dólares. Considerando la tendencia de esta variable para diversas categorías de empresas se identifican seis hechos estilizados principales:

1. Después de la crisis de 1995, la empresa promedio y mediana experimentó un aumento en la participación de su deuda en dólares. - Los resultados del modelo desarrollado en la sección 2 sugieren que después de la crisis, los acreedores (y también las empresas) han dado mayor ponderación al riesgo del tipo de cambio. Como se mencionó, esto pudo haber sucedido debido a que, antes de 1995, se esperaba que el gobierno otorgara un seguro implícito contra movimientos en cambiarios. La implicación más obvia de 
esta hipótesis sería una reducción de la participación de la deuda en dólares sobre la deuda total. Sin embargo, los datos en las dos primeras filas del Cuadro 2 muestran que la participación de la deuda en dólares sobre la deuda total aumentó súbitamente de 1992 a 1994, de 25\% a 32\%. Después de la crisis, en 1995, el nivel aumentó significativamente, como era de esperarse después de una devaluación. Sin embargo, a pesar de que éste decreció en 1999 y en 2000 a un nivel de 42\%, siguió por arriba del nivel previo a la crisis. Esta tendencia es muy similar si tomamos solamente a las empresas con deuda en dólares. Ello implica que el aumento mencionado no puede ser explicado por una mayor proporción de empresas con deuda denominada en moneda extranjera.

Los hechos que se presentan a continuación indican que hubo varios factores que contribuyeron al aumento del endeudamiento relativo en dólares y los cuales son compatibles con los resultados de nuestro modelo.

2. La deuda en dólares aumentó más para las empresas exportadoras. - Las altas tasas de crecimiento experimentadas por las exportaciones a partir de 1995, permitieron un mayor endeudamiento en dólares de las empresas sin aumentar el riesgo cambiario. El cociente de las exportaciones respecto a las ventas aumentó de $1 \%$ en 1994 a $6 \%$ en 2000 para toda la muestra y aumentó de $2 \%$ a $8 \%$ para las empresas con deuda en dólares (Cuadro 2). También, como se mencionó, en este período más empresas se volvieron exportadoras. En 1992, 58\% de las empresas en la muestra eran exportadoras mientras que $68 \%$ lo eran en 2000.

Al considerar la deuda en dólares de las empresas exportadoras contra la de las noexportadoras, se explica, al menos parcialmente, el incremento de la importancia de la deuda en dólares a la vez que disminuía la exposición al riesgo cambiario. Los datos del Cuadro 2 muestran que la participación de la deuda en dólares para las empresas exportadoras cambió de $52 \%$ a 58\% durante el período 1994-2000, mientras que para las no-exportadoras siguió manteniéndose constante en un nivel de $8 \%$.

Incluso aún cuando en el período de 1992-1994 a 2000 no se aprecia una disminución de la deuda en dólares en las empresas no-exportadoras, eso se debe a que su deuda 
total disminuyó drásticamente. Para las empresas no-exportadoras la deuda total sobre los activos disminuyó en ese lapso de $41 \%$ a $28 \%$, mientras que para las exportadoras aumentó marginalmente de $44 \%$ a $46 \%$ (Cuadro 2). Retomaremos este punto más adelante.

3. Las empresas pequeñas y medianas aumentaron su endeudamiento en dólares ${ }^{6 /}$ - - De 1992 a 1994, estas empresas, pero especialmente las pequeñas tuvieron una proporción muy baja de deuda en dólares sobre la deuda total. Como se muestra en el Cuadro 2, durante ese período esta proporción permaneció en proximadamente $4 \%$ para las empresas pequeñas y en aproximadamente $31 \%$ para las medianas. La proporción de deuda en dólares sobre el total para las empresas medianas experimentó un aumento significativo antes de 1992, debido probablemente a la liberalización financiera, aunque después se mantuvo estable. Sin embargo, después de la modificación del régimen cambiario, dicha proporción siguió una tendencia creciente que alcanzó niveles respectivos de $19 \%$ y $42 \%$ en 2000 para las empresas pequeñas y medianas.

4. Las empresas más grandes mantuvieron una mayor proporción de deuda en dólares, pero esa proporción disminuyó después de la crisis. - Se ha argumentado, y probado empíricamente en la literatura, que en México las empresas más grandes tienen mayor acceso al crédito porque, entre otras cosas, cuentan con activos con un mayor valor que podría utilizarse como colateral (Babatz, 1997 y Gelos y Werner, 1999). Sin embargo, también se ha encontrado en otros países y con datos de empresas públicas, que la inversión de las empresas grandes ha sido más sensible a los flujos de liquidez, indicando que esas empresas enfrentan mayores restricciones de crédito (Devereux y Schiantarelli, 1990). Además, Olin y Rudebusch (1992) han encontrado que no hay diferencias significativas entre los grupos de tamaño para las empresas listadas en el NYSE.

\footnotetext{
${ }^{6 /}$ En el documento se define al tamaño de una empresa de acuerdo con el valor de sus activos. Para cada año se dividió la muestra en cuartiles, y el primer cuartil corresponde a las empresas pequeñas, el segundo y tercer cuartiles a las empresas medianas y el cuarto a las grandes. Debe quedar claro que este proceso no obliga a que una empresa se mantenga en la misma categoría dentro de la muestra.
} 


\section{Cuadro 2}

(medianas)

\begin{tabular}{|c|c|c|c|c|c|c|c|c|c|}
\hline & 1992 & 1993 & $1994 *$ & 1995 & 1996 & 1997 & 1998 & 1999 & 2000 \\
\hline \multicolumn{10}{|c|}{ Deuda en USD / Deuda total } \\
\hline Todas & $25 \%$ & $30 \%$ & $32 \%$ & 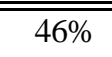 & $44 \%$ & "44\% & 46\% & 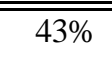 & $\overline{42 \%}$ \\
\hline Deuda en USD & $33 \%$ & $37 \%$ & $39 \%$ & $52 \%$ & $52 \%$ & $52 \%$ & $55 \%$ & $49 \%$ & $52 \%$ \\
\hline No Exportadoras & $43 \%$ & $45 \%$ & $52 \%$ & $62 \%$ & $55 \%$ & $60 \%$ & $63 \%$ & $55 \%$ & $58 \%$ \\
\hline Exportadoras & $3 \%$ & $3 \%$ & $8 \%$ & $11 \%$ & $12 \%$ & $8 \%$ & $11 \%$ & $9 \%$ & $8 \%$ \\
\hline Empresas Pequeñas & $5 \%$ & $4 \%$ & $5 \%$ & $11 \%$ & $27 \%$ & $20 \%$ & $13 \%$ & $18 \%$ & $19 \%$ \\
\hline Empresas Medianas & $28 \%$ & $31 \%$ & $34 \%$ & $45 \%$ & $38 \%$ & $41 \%$ & $43 \%$ & $42 \%$ & $42 \%$ \\
\hline Empresas Grandes & $55 \%$ & $60 \%$ & $64 \%$ & $78 \%$ & $74 \%$ & $76 \%$ & $80 \%$ & $64 \%$ & $63 \%$ \\
\hline \multicolumn{10}{|c|}{ Exportaciones / Ventas Totales } \\
\hline Todas & $1.2 \%$ & $0.6 \%$ & $1.0 \%$ & $3.5 \%$ & $5.2 \%$ & $5.8 \%$ & $5.2 \%$ & $5.6 \%$ & $6.3 \%$ \\
\hline Deuda en USD & $2.5 \%$ & $1.7 \%$ & $2.0 \%$ & $6.1 \%$ & $7.0 \%$ & $7.7 \%$ & $7.4 \%$ & $7.4 \%$ & $8.2 \%$ \\
\hline Sin Deuda en USD & $0.0 \%$ & $0.0 \%$ & $0.0 \%$ & $0.0 \%$ & $0.0 \%$ & $0.0 \%$ & $0.0 \%$ & $0.0 \%$ & $0.0 \%$ \\
\hline Exportadoras & $7.1 \%$ & $6.8 \%$ & $6.6 \%$ & $18.9 \%$ & $15.5 \%$ & $14.2 \%$ & $15.1 \%$ & $13.6 \%$ & $17.4 \%$ \\
\hline Empresas Pequeñas & $0.0 \%$ & $0.0 \%$ & $0.0 \%$ & $0.0 \%$ & $2.4 \%$ & $1.3 \%$ & $0.3 \%$ & $0.9 \%$ & $0.5 \%$ \\
\hline Empresas Medianas & $1.5 \%$ & $0.8 \%$ & $1.1 \%$ & $2.5 \%$ & $3.5 \%$ & $4.8 \%$ & $4.7 \%$ & $3.7 \%$ & $3.7 \%$ \\
\hline Empresas Grandes & $4.7 \%$ & $2.9 \%$ & $4.8 \%$ & $18.9 \%$ & $18.5 \%$ & $17.5 \%$ & $20.4 \%$ & $14.1 \%$ & $18.2 \%$ \\
\hline \multicolumn{10}{|c|}{ Deuda Total / Activos } \\
\hline Todas & $41 \%$ & $42 \%$ & $43 \%$ & $47 \%$ & $44 \%$ & $\overline{43 \%}$ & $41 \%$ & $42 \%$ & $\overline{43 \%}$ \\
\hline Deuda en USD & $43 \%$ & $42 \%$ & $44 \%$ & $48 \%$ & $45 \%$ & $43 \%$ & $42 \%$ & $43 \%$ & $45 \%$ \\
\hline Sin Deuda en USD & $40 \%$ & $40 \%$ & $34 \%$ & $37 \%$ & $38 \%$ & $29 \%$ & $34 \%$ & $35 \%$ & $27 \%$ \\
\hline Exportadoras & $43 \%$ & $42 \%$ & $44 \%$ & $49 \%$ & $46 \%$ & $44 \%$ & $47 \%$ & $46 \%$ & $46 \%$ \\
\hline No Exportadoras & $41 \%$ & $40 \%$ & $41 \%$ & $44 \%$ & $38 \%$ & $32 \%$ & $29 \%$ & $33 \%$ & $28 \%$ \\
\hline Empresas Pequeñas & $46 \%$ & $47 \%$ & $46 \%$ & $48 \%$ & $43 \%$ & $39 \%$ & $34 \%$ & $36 \%$ & $38 \%$ \\
\hline Empresas Medianas & $39 \%$ & $40 \%$ & $42 \%$ & $45 \%$ & $42 \%$ & $42 \%$ & $42 \%$ & $41 \%$ & $43 \%$ \\
\hline Empresas Grandes & $37 \%$ & $41 \%$ & $44 \%$ & $46 \%$ & $45 \%$ & $45 \%$ & $47 \%$ & $46 \%$ & $48 \%$ \\
\hline \multicolumn{10}{|c|}{ Exportaciones / Deuda en USD } \\
\hline Todas & $4.0 \%$ & $2.1 \%$ & $2.0 \%$ & $6.3 \%$ & $6.9 \%$ & $8.1 \%$ & 6.4\% & $7.4 \%$ & $\overline{7.4 \%}$ \\
\hline Exportadoras & $9.0 \%$ & $7.5 \%$ & $8.1 \%$ & $11.3 \%$ & $11.9 \%$ & $14.8 \%$ & $12.3 \%$ & $12.5 \%$ & $13.7 \%$ \\
\hline Empresas Pequeñas & $0.6 \%$ & $0.0 \%$ & $0.0 \%$ & $2.7 \%$ & $8.6 \%$ & $7.3 \%$ & $4.7 \%$ & $11.6 \%$ & $11.6 \%$ \\
\hline Empresas Medianas & $7.2 \%$ & $3.2 \%$ & $2.0 \%$ & $3.2 \%$ & $5.2 \%$ & $6.2 \%$ & $4.9 \%$ & $6.8 \%$ & $7.0 \%$ \\
\hline Empresas Grandes & $3.9 \%$ & $2.1 \%$ & $2.7 \%$ & $9.5 \%$ & $10.5 \%$ & $9.4 \%$ & $8.6 \%$ & $7.7 \%$ & $8.3 \%$ \\
\hline \multicolumn{10}{|c|}{ Número de Empresas } \\
\hline$\overline{\text { Todas }}$ & $\overline{260}$ & 253 & 237 & 224 & 220 & 209 & 186 & 169 & 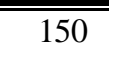 \\
\hline Deuda en USD & 215 & 213 & 207 & 197 & 195 & 188 & 165 & 150 & 134 \\
\hline Sin Deuda en USD & 45 & 39 & 29 & 26 & 24 & 21 & 21 & 19 & 16 \\
\hline Exportadoras & 151 & 148 & 139 & 144 & 148 & 140 & 125 & 120 & 103 \\
\hline No Exportadoras & 105 & 103 & 96 & 79 & 70 & 67 & 60 & 49 & 45 \\
\hline Empresas Pequeñas & 65 & 64 & 60 & 56 & 55 & 53 & 47 & 43 & 38 \\
\hline Empresas Medianas & 130 & 126 & 118 & 112 & 110 & 104 & 93 & 84 & 75 \\
\hline Empresas Grandes & 65 & 63 & 59 & 56 & 55 & 52 & 46 & 42 & 37 \\
\hline
\end{tabular}

* Medianas de los primeros tres trimestres.

Bajo el supuesto de que el problema de información asimétrica es más severo para los acreedores extranjeros que para los internos, y mayor para las empresas pequeñas que 
para las grandes, este último grupo mantendría un mayor endeudamiento en dólares que los anteriores. En el Cuadro 2 se aprecia en efecto que las empresas grandes son las que han tenido una mayor proporción de deuda en dólares, seguidas, en primer lugar, por las medianas y luego por las pequeñas. Más aún, estas firmas experimentaron el mayor crecimiento de 1990 a 1994.

Aún más interesante es la forma de "U" invertida que siguió durante los noventas la participación de la deuda en dólares para las empresas grandes. Es decir, después de la crisis y especialmente desde 1998 dicha proporción disminuyó para las empresas grandes mientras que aumentó para las empresas medianas y pequeñas. La proporción era de 64\% para las empresas grandes en 1994 y aumentó hasta 78\% en 1996 para luego caer otra vez hasta $63 \%$ en 2000 . En la siguiente sección volveremos a este tema al analizar la validez empírica del modelo propuesto para explicar este comportamiento.

5. La exposición en dólares disminuyó después de la crisis. - Los hechos descritos indican que después de 1996 las empresas exportadoras han aumentado su endeudamiento relativo en dólares mientras que las empresas no-exportadoras lo han mantenido en niveles relativamente estables. Como las exportaciones aumentaron significativamente en este período, es importante analizar qué sucedió con la exposición de las empresas, medida como la relación entre las exportaciones y la deuda en dólares. Esta relación también controla el efecto de la devaluación que contribuyó al aumento del endeudamiento en dólares en términos de la deuda total, en ausencia de nuevos créditos. Antes de pasar a la estimación formal, se considera la relación entre las exportaciones y la deuda en dólares. Este análisis revela que dicho cociente aumentó de 4\% en 1992 a 7\% en 2000 (Cuadro 2). Por tanto, el aumento de las exportaciones contrarrestó el aumento del endeudamiento en dólares, de manera que aun si tomamos 1992 como el año base las empresas mantuvieron una menor exposición al riesgo cambiario que antes. Al dividir la mue stra por tamaño de empresa, este resultado subsiste. Incluso las empresas medianas siguen siendo las más expuestas. Así, la relación entre las exportaciones totales y la deuda en dólares aumentó de 2\% en 1992 a casi 7\% en 2000. Tampoco es sorprendente que en 1994 las empresas en todas las categorías alcanzaran la exposición más alta del período. 
6. De $1994^{7 /}$ a 2000 la deuda total como proporción de los activos disminuyó para las empresas con un bajo o nulo endeudamiento en dólares. - Desde 1995 y casi hasta 2000 el crédito otorgado por los bancos mexicanos fue muy restringido, implicando que el aumento en la importancia de la deuda en dólares es explicado parcialmente por la reducción de la deuda en pesos. Como puede verse en el Cuadro 2, la relación entre la deuda total y los activos totales disminuyó dramáticamente para las empresas con una baja proporción de deuda en dólares. Para las empresas sin deuda en dólares la mediana de esta relación cayó de 34\% en 1994 a 27\% en 2000, para las empresas noexportadoras de $41 \%$ a $28 \%$ y para las pequeñas de $46 \%$ a $38 \%$. Para el resto de las empresas la relación sólo aumentó marginalmente y definitivamente menos que lo que se podría esperar por la depreciación del peso ocurrida en 1994.

Todos estos resultados indican no sólo que las empresas más pequeñas podrían haber tenido más acceso al crédito en dólares (incluso cuando la deuda total disminuyó como proporción de los activos totales para estas empresas) después de la crisis, sino que dado el crecimiento vigoroso de las exportaciones se volvieron menos vulnerables. Por otro lado, las empresas más grandes, que son también las que exportan más y las que alcanzaron el crecimiento más alto de ventas al exterior, han reducido marginalmente su deuda en dólares. Esto, en la medida en que eran las que tenían mayor endeudamiento antes de 1994.

\section{III.2. Análisis econométrico}

Una vez que se abandona el mundo de mercados perfectos de Miller y Modigliani, donde la estructura de capital se determina aleatoriamente y el costo de diversas fuentes del financiamiento es el mismo, es difícil encontrar una explicación sencilla para los determinantes de la estructura de deuda y capital. Esto se debe a que la información asimétrica se presenta de maneras muy diversas, por lo que varios contratos podrían solucionar los problemas de incentivos. Más aún, respecto al problema específico de información descrito en el modelo desarrollado en la sección 2, hay costos de transacción que difieren entre las diversas fuentes de financiamiento y entre las empresas. Sin embargo, ese modelo es suficientemente rico para permitirnos hacer un buen "mapeo" de las

\footnotetext{
${ }^{7 /}$ Considerando el nivel de la mediana del año hasta el tercer trimestre.
} 
variables usadas en nuestra aplicación empírica a los parámetros del modelo. De ahí pasamos a probar nuestra hipótesis de que bajo un régimen de tipo de cambio flexible, los acreedores internalizan en mayor medida el riesgo cambiario que bajo un régimen de tipo de cambio predeterminado.

Antes de adentrarnos en las especificaciones econométricas, es importante asegurar que un supuesto fundamental del modelo se cumpla. La tasa de interés externa ajustada por las expectativas de devaluación debe ser menor que la tasa de interés local. En la Gráfica 1 se muestra que durante el período de análisis la tasa de interés del exterior (tomada como la tasa activa en EU más la devaluación esperada implícita en los mercados a futuro y el riesgo país dado por el diferencial entre la tasa de los bonos mexicanos gubernamentales denominados en dólares menos la tasa de los bonos a 30 años del Tesoro de EUA) fue siempre menor que la tasa activa en México.

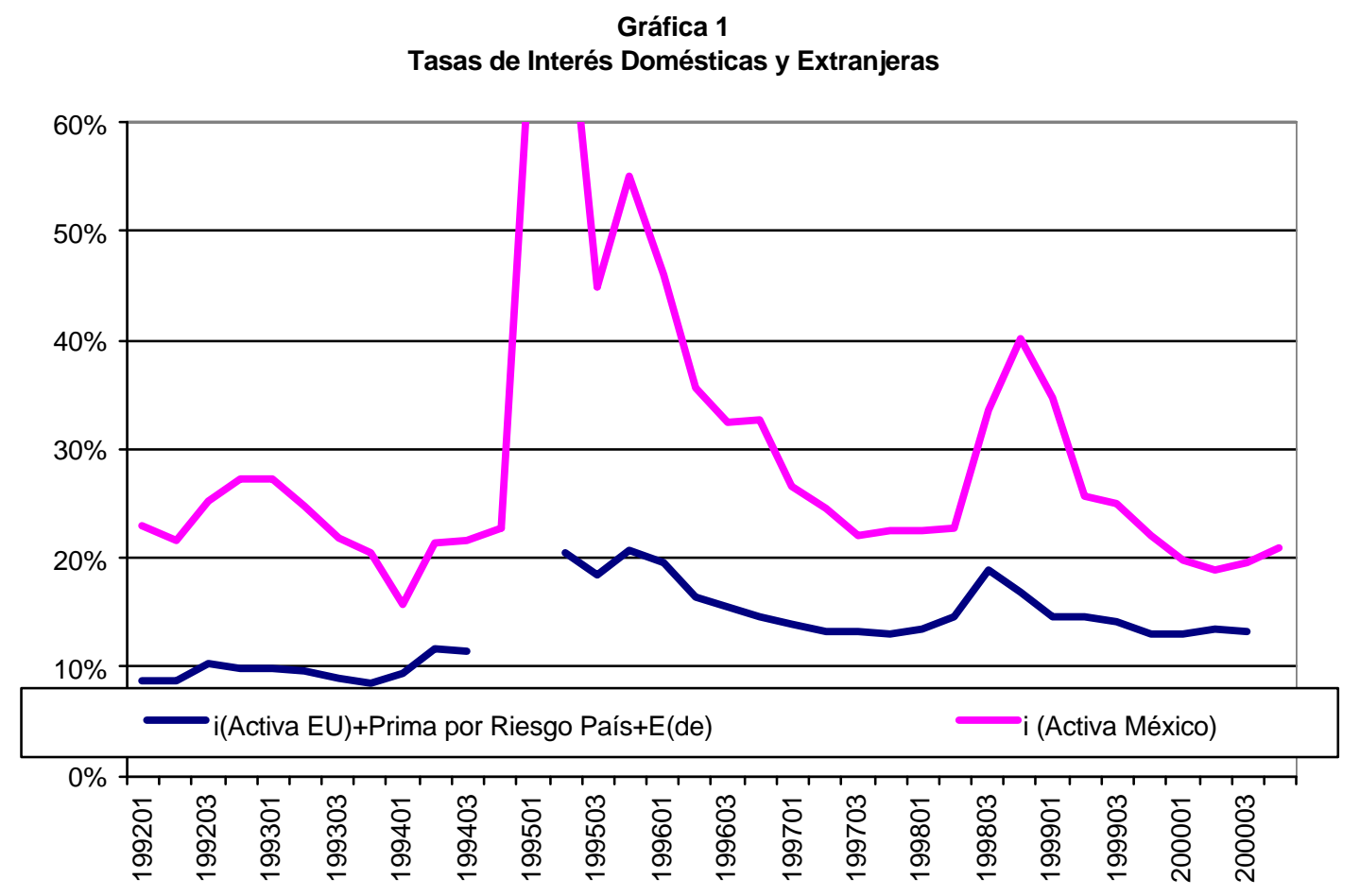

Con base en el marco teórico presentado en la sección 2, la finalidad es comparar los coeficientes asociados con las ventas internas y las exportaciones, ambas como proporción de la deuda total, en una regresión que tiene como variable endógena a la deuda en dólares 
como proporción de la total. Con este fin se adoptaron dos tipos de especificaciones. En la primera, se supone que todos los recursos externos provienen ya sea de bancos locales o de deuda extranjera, reflejando la expresión derivada en la sección 2 como la ecuación (8). Es decir, no se consideraron fuentes adicionales de financiamiento como la posibilidad de emitir deuda corporativa en pesos o acciones. En una segunda especificación a la deuda total se le sumaron las acciones en manos de accionistas minoritarios. Adicionalmente, se abrió la posibilidad de tener deuda corporativa interna, la cual difiere de la bancaria debido a que los acreedores no realizan ningún tipo de supervisión sobre el deudor. La derivación de esta nueva ecuación es directa. Ello, en razón de que este tipo de financiamiento es menos costoso que el crédito bancario, pero más que la deuda en dólares. La nueva ecuación (8) sería:

$$
\frac{I_{u} E_{t}}{T E R}=\underbrace{\frac{P_{H}}{r^{*}}}_{\alpha} \frac{\operatorname{Exp}}{T E R}+\underbrace{\frac{P_{H}}{r^{*}} \frac{E_{t}}{E_{t+1}}}_{\beta}\left[\frac{\text { Vtas Domest Netas }}{T E R}-\frac{(c+b) / \Delta P}{T E R}-\frac{M E+C I}{T E R}\right]
$$

Donde:

TER Recursos externos totales de la empresa, o deuda total más acciones en manos de accionistas minoritarios.

ME Acciones en manos de accionistas minoritarios.

CI Deuda corporativa interna.

La estimación de (8') requiere de datos que separen la deuda en pesos en bancaria y corporativa, pero como no tenemos esa información suponemos que esta composición es la misma que la observada en la deuda total.

Para controlar por la tecnología de supervisión o por los beneficios privados que se pueden obtener, incluimos dos variables dicotómicas. La primera de ellas para empresas que han emitido ADRs y la segunda para empresas tenedoras o holdings. Los activos totales también se añadieron como una "proxy" para el tamaño, lo que puede también afectar los parámetros: $c$, $b$ o $P_{H}$ del modelo. 
Debido a que algunas de las variables utilizadas no coinciden exactamente con las consideradas en el modelo, es importante verificar que cumplan con los supuestos implícitos. Dadas las estrictas limitaciones al otorgamiento de créditos en moneda extranjera en México, la deuda en dólares es casi igual al crédito concedido por los bancos extranjeros más la deuda emitida fuera de México ${ }^{8 /}$. En ambos casos, la supervisión de las empresas mexicanas es más cara que para los bancos locales, satisfaciendo los supuestos del modelo. Los datos confirman además el supuesto de que el crédito interno es a corto plazo mientras que el crédito extranjero tiene en general un plazo más largo. El 84\% del crédito local concedido en 1999 tuvo un plazo igual o menor a un año, y el 91\% para 2000. Por otro lado, durante 1999 la deuda en dólares tuvo un vencimiento mayor que un año en el $75 \%$ de los casos y en el $66 \%$ durante 2000 .

La variable endógena está dada por la relación entre la deuda en dólares y la deuda total al final de cada período. Además de las variables exógenas mencionadas se incluye el nivel inicial de la variable endógena. El resto de las variables exógenas se considera para el año inicial de cada período. Con el propósito de analizar si se dio un cambio en los determinantes del crédito en dólares después de la adopción del régimen de flotación del tipo de cambio se dividió la muestra en dos períodos. Para el primero (segundo) período se tomaron las variables exógenas para 1992 (1996) y las endógenas para 1994 (2000). Se excluyó 1995 para evitar algún efecto asociado a la volatilidad experimentada durante la crisis. Considerando que la variable endógena está restringida al intervalo $(0,1)$ se emplea un modelo Tobit para la estimación de la siguiente ecuación:

$U S D D_{i}^{T}=\alpha_{0}+\alpha_{1} U S D D_{i}+\alpha_{2} X_{i}^{t}+\alpha_{3 D} D S_{i}^{t}+\alpha_{4}$ Tamaño $_{i}^{t}+\alpha_{5} A D R_{i}+\alpha_{6} H_{i}+\alpha_{6} M E_{i}+\alpha_{6} C I_{i}+\varepsilon_{i}^{t}$

Es importante recordar que, como se utilizó la relación entre la deuda en dólares y la deuda total, resolvimos, al menos parcialmente, la crítica potencial de una correlación espuria. Esta crítica pudiera ser relevante porque al no controlar por oportunidades de inversión, se podría argumentar que los resultados se debieran a que durante el primer período estas

\footnotetext{
${ }^{8 /}$ Usando información de 1999 y 2000 obtenemos respectivamente que un $85 \%$ y un $77 \%$ de la deuda en
} 
oportunidades tenían una relación positiva con el tamaño de la empresa mientras que en el segundo período este potencial estuvo más relacionado con la categoría de empresas exportadoras. Sin embargo, debido a que una mayor proporción de deuda en dólares no necesariamente implica una mayor deuda total, esta crítica carece de validez. Lo anterior es evidente en el Cuadro 2, donde puede verse que la deuda total como proporción de los activos no mostró el mismo patrón que la deuda en dólares.

Este punto se vuelve aún más relevante si consideramos que la firma del Tratado de Libre Comercio de América del Norte (TLCAN) en 1994 pudo haber cambiado las oportunidades de financiamiento para las empresas exportadoras ya que éstas podrían haber sido consideradas como las empresas con mayor potencial de crecimiento. Harris et al (1994) encontraron que después de la liberalización financiera en Indonesia, las condiciones crediticias para las empresas exportadoras mejoraron significativamente. Por tanto, aunque la liberalización comercial y financiera en México ocurrió anteriormente, en 1987 y 1991 respectivamente, el TLCAN ofreció nuevas oportunidades a algunas empresas. Bajo estas circunstancias, las empresas exportadoras pudieron haber conseguido un mayor financiamiento en el período posterior a la crisis no porque implicara un riesgo cambiario menor, sino por el potencial de crecimiento que tenían. Además, si el endeudamiento en dólares es la única fuente de financiamiento marginal (como es el caso en el modelo de la sección 2), su participación debería estar correlacionada con los beneficios.

Para ahondar en este tema incluimos en las regresiones el crecimiento medio anual de las ventas de 1992 a 1994 para el primer período y de 1996 a 2000 para el segundo. Ello a fin de controlar por las oportunidades de inversión. El Cuadro 3 muestra los resultados, donde las columnas (I) corresponden al primer período y las columnas (II) al segundo. En el último renglón del cuadro se reportan los estadísticos-F correspondientes a la prueba de igualdad del coeficiente de ventas internas y el de ventas al exterior. De acuerdo con nuestra hipótesis, esperamos que no sean significativamente diferentes para el primer período, pero que difieran en el segundo. Las primeras cuatro columnas presentan los resultados considerando sólo la deuda total y las últimas cuatro considerando también

dólares fue emitida en EU o extendida por bancos extranjeros ubicados fuera de México. 
dentro de los recursos externos totales a los accionistas minoritarios así como a la deuda corporativa $^{9 /}$.

Por lo que respecta al resto de las variables, los resultados son muy similares para las ocho especificaciones. La variable de tamaño siempre resultó significativa para el primer período pero nunca para el segundo, mientras que las exportaciones siempre son significativas para el segundo período, pero nunca para el primero. El resto de las variables (crecimiento de las ventas, conglomerados o ADRs) nunca fueron significativas.

Estos resultados pueden implicar una conclusión muy interesante. Antes de la crisis el principal determinante de la participación de la deuda en dólares era el tamaño de la empresa. Ello puede indicar que los acreedores extranjeros sólo se preocupaban por esa variable probablemente por la existencia de rendimientos crecientes en la supervisión, por lo que era más barato dar seguimiento a las empresas mayores. En términos de la importancia otorgada al riesgo cambiario durante el primer período, podemos concluir que hubo muy poca diferencia entre las ventas internas y las externas. Tal como se muestra en la prueba $\mathrm{F}$, los coeficientes de esas dos variables no son significativamente diferentes. Sin embargo, para el segundo período la única variable significativa la constituyen las exportaciones. Esto sugiere que para los acreedores (y probablemente también para los deudores) la exposición al tipo de cambio es tan importante, que sólo se preocupan por los beneficios en dólares y no por los totales. En este caso, la prueba $\mathrm{F}$ indica que las ventas al extranjero y las internas tienen coeficientes significativamente diferentes. El coeficiente para los accionistas minoritarios resultó negativo y significativo como lo predice el modelo en sólo una especificación. Por su parte, la deuda corporativa en pesos nunca fue significativa.

\footnotetext{
${ }^{9 /}$ Utilizamos una especificación similar para estimar la ecuación derivada de la relación entre deuda y capital para realizar una prueba adicional de nuestra teoría. Los resultados de estos ejercicios no se incluyen, pero encontramos que ninguna de las variables explicativas fue significativa en ninguno de los períodos. Una posible explicación sería que el error en la medición es más severo para el capital que para la deuda, en parte porque las cifras históricas están únicamente ajustadas por la inflación y no por cambios en el valor de mercado. También debido a que las diferencias entre el valor histórico y el valor de mercado tienden a ser más importantes para las acciones que para la deuda.
} 


\section{Cuadro 3}

Determinantes de la Deuda en Dólares

\begin{tabular}{|c|c|c|c|c|c|c|c|c|}
\hline & \multicolumn{2}{|c|}{$(1)$} & \multicolumn{2}{|c|}{ (2) } & \multicolumn{2}{|c|}{ (3) } & \multicolumn{2}{|c|}{ (4) } \\
\hline & I & II & I & II & I & II & I & II \\
\hline Constante & $\begin{array}{c}-0.34^{* * *} \\
(0.12)\end{array}$ & $\begin{array}{c}-0.05 \\
(0.16)\end{array}$ & $\begin{array}{c}-0.20^{* * * *} \\
(0.14)\end{array}$ & $\begin{array}{c}0.02 \\
(0.19)\end{array}$ & $\begin{array}{r}-0.41 \text { *** } \\
(0.13)\end{array}$ & $\begin{array}{c}0.06 \\
(0.18)\end{array}$ & $\begin{array}{c}-0.19 \\
(0.14)\end{array}$ & $\begin{array}{c}0.02 \\
(0.21)\end{array}$ \\
\hline Deuda en dlls/ D euda Total Rezagada & $\begin{array}{c}0.78 * * * \\
(0.06)\end{array}$ & $\begin{array}{c}0.74 * * * \\
(0.07)\end{array}$ & $\begin{array}{c}0.84^{* * * *} \\
(0.06)\end{array}$ & $\begin{array}{c}0.75^{* * * *} \\
(0.07)\end{array}$ & $\begin{array}{r}0.76^{* * *} \\
(0.06)\end{array}$ & $\begin{array}{c}0.74^{* * * *} \\
(0.07)\end{array}$ & $\begin{array}{c}0.84^{* * *} \\
(0.07)\end{array}$ & $\begin{array}{c}0.77 * * * \\
(0.05)\end{array}$ \\
\hline Log de Activos Totales & $\begin{array}{c}0.04 * * * \\
(0.01)\end{array}$ & $\begin{array}{c}0.01 \\
(0.01)\end{array}$ & $\begin{array}{l}0.03 * * \\
(0.01)\end{array}$ & $\begin{array}{c}0.00 \\
(0.01)\end{array}$ & $\begin{array}{r}0.04 * * * \\
(0.01)\end{array}$ & $\begin{array}{l}-0.002 \\
(0.01)\end{array}$ & $\begin{array}{c}0.03 \\
(0.01)\end{array}$ & $\begin{array}{c}-0.001 \\
(0.02)\end{array}$ \\
\hline Exportaciones/ DeudaTotal & $\begin{array}{c}0.11 \\
(0.18)\end{array}$ & $\begin{array}{c}0.37 * * * \\
(0.12)\end{array}$ & $\begin{array}{c}0.06 \\
(0.18)\end{array}$ & $\begin{array}{c}0.39 * * * \\
(0.17)\end{array}$ & $\begin{array}{c}0.13 \\
(0.17)\end{array}$ & $\begin{array}{c}0.34^{* * * *} \\
(0.13)\end{array}$ & $\begin{array}{c}0.03 \\
(0.01)\end{array}$ & $\begin{array}{l}0.31^{*} \\
(0.18)\end{array}$ \\
\hline Ventas Totales/ D euda Total & $\begin{array}{c}-0.10 \\
(0.09)\end{array}$ & $\begin{array}{c}-0.05 \\
(0.07)\end{array}$ & $\begin{array}{c}-0.08 \\
(0.09)\end{array}$ & $\begin{array}{c}-0.170 \\
(0.13)\end{array}$ & $\begin{array}{c}-0.11 \\
(0.09)\end{array}$ & $\begin{array}{l}-0.005 \\
(0.08)\end{array}$ & $\begin{array}{c}-0.06 \\
(0.09)\end{array}$ & $\begin{array}{c}-0.180 \\
(0.15)\end{array}$ \\
\hline Accionistas Minoritarios & & & & & $\begin{array}{c}-0.23^{* *} \\
(0.11)\end{array}$ & $\begin{array}{l}0.030 \\
(0.16)\end{array}$ & $\begin{array}{l}-0.06 \\
(0.09)\end{array}$ & $\begin{array}{c}0.12 \\
(0.17)\end{array}$ \\
\hline D euda Corporativa en Pesos & & & & & $\begin{array}{c}0.00 \\
(0.00)\end{array}$ & $\begin{array}{l}0.000 \\
(0.00)\end{array}$ & $\begin{array}{c}0.00 \\
(0.00)\end{array}$ & $\begin{array}{c}0.00 \\
(0.00)\end{array}$ \\
\hline Tasa Media de Crecimiento de Vtas & & & $\begin{array}{c}-0.03 \\
(0.07)\end{array}$ & $\begin{array}{c}0.00 \\
(0.00)\end{array}$ & & & $\begin{array}{c}-0.05 \\
(0.07)\end{array}$ & $\begin{array}{l}0.120 \\
(0.08)\end{array}$ \\
\hline Dummy de ADR's & & & $\begin{array}{c}-0.04 \\
(0.09)\end{array}$ & $\begin{array}{c}0.04 \\
(0.05)\end{array}$ & & & $\begin{array}{l}-0.03 \\
(0.09)\end{array}$ & $\begin{array}{c}0.00 \\
(0.05)\end{array}$ \\
\hline Dummy de Holdings & & & $\begin{array}{c}0.01 \\
(0.04)\end{array}$ & $\begin{array}{c}0.08 \\
(0.05)\end{array}$ & & & $\begin{array}{c}0.02 \\
(0.04)\end{array}$ & $\begin{array}{c}0.04 \\
(0.06)\end{array}$ \\
\hline Tamaño de la Muetra & 209 & 137 & 185 & 115 & 208 & 135 & 184 & 113 \\
\hline LR Chi ${ }^{2}$ & 211.69 & 137.79 & 197.13 & 135.98 & 207.1 & 115.18 & 190.29 & 113.5 \\
\hline Prob $>$ Chi $^{2}$ & 0.00 & 0.00 & 0.00 & 0.00 & 0.00 & 0.00 & 0.00 & 0.00 \\
\hline Pseudo R 2 & 1.33 & 1.14 & 1.44 & 1.29 & 1.55 & 1.20 & 1.71 & 1.40 \\
\hline $\begin{array}{l}F \\
\text { Prob }>F\end{array}$ & $\begin{array}{l}0.98 \\
0.32\end{array}$ & $\begin{array}{l}8.29 \\
0.00\end{array}$ & $\begin{array}{l}0.41 \\
0.52\end{array}$ & $\begin{array}{l}5.65 \\
0.02\end{array}$ & $\begin{array}{l}1.39 \\
0.24\end{array}$ & $\begin{array}{l}4.92 \\
0.03\end{array}$ & $\begin{array}{l}0.18 \\
0.67\end{array}$ & $\begin{array}{r}3.99 \\
0.048\end{array}$ \\
\hline $\begin{array}{l}\text { La variable dependiente es D euda en D } \\
\text { D esviación Estándar en Paréntesis } \\
\text { I: 1994-variable endógena y 1992-variab } \\
\text { D ummy de AD R's=1 desde la primera } \\
\text { * Significativo al 10\% } \\
\text { ** Significativo al } 5 \% \\
\text { *** Significativo al } 1 \%\end{array}$ & $\begin{array}{l}\text { euda } \mathrm{To} \\
\text { xógenas. } \\
\text { a de } \mathrm{AD}\end{array}$ & $\begin{array}{l}\text { II: 2000- } \\
\text { Rs o bono }\end{array}$ & $\begin{array}{l}\text { variable en } \\
\text { s en EU. }\end{array}$ & dógena 1 & 96-vari & les exóc & & \\
\hline
\end{tabular}


Es importante notar que los acreedores pueden haber actuado racionalmente en ambos períodos dadas las expectativas respecto del comportamiento del gobierno. Desafortunadamente, con nuestros datos no es posible distinguir la razón que motivó este cambio en las expectativas. Por un lado, el régimen de tipo de cambio fijo dio a las empresas y a los bancos un seguro implícito por parte del gobierno ante movimientos del tipo de cambio. Por otra parte, con el régimen de tipo de cambio flexible estos subsidios quedaron eliminados. Antes de 1994 era difícil imaginar una crisis de tal magnitud, por lo que nadie consideró tal escenario como posible. Es decir, más que la modificación de régimen cambiario, fue la posibilidad asignada al estallido de una gran crisis lo que se vio afectada. Para probar estas hipótesis alternativas, se podrían comparar estos resultados de México con los de otros países, como Argentina o Brasil (antes de 1999) en esos años. Aunque dichos países no experimentaron una crisis tan aguda, también sufrieron presiones especulativas sobre sus tipos de cambio. De ahí que las tasas de interés se incrementaran rápidamente y enfrentaran una importante recesión, pero conservando sus regímenes cambiarios predeterminados.

A continuación usamos los coeficientes estimados para separar el cambio de la participación de la deuda en dólares de 1994 a $2000\left(\widehat{U S D} D_{00}-U \widehat{S D} D_{94}\right)$ en tres componentes:

1. El efecto atribuible a las disparidades entre los niveles de largo plazo para cada período, el cual a su vez puede descomponerse en:

- El efecto atribuible al cambio en los determinantes del crédito o en los coeficientes de la regresión y

- El efecto atribuible a cambios en las características de la empresa promedio.

2. El efecto atribuible a las disparidades entre el nivel de corto plazo, dado un nivel inicial de deuda en dólares, y el nivel de largo plazo hacia el que esta relación estaba convergiendo en ambos períodos. 


$$
\begin{aligned}
& U \widehat{S D} D_{00}-U \widehat{S D} D_{94}=U \widehat{S D} D_{L P}^{2}-U \widehat{S D D^{1}}{ }_{L P}+\underbrace{\left(U \widehat{S D D_{00}}-U \widehat{S D} D^{2}{ }_{L P}\right)-\left(U S \widehat{D D} D_{94}-U \widehat{S D} D_{L P}\right)}_{\text {Disparidadentre losnivelesde corto plazo y largoplazo }} \\
& U S \widehat{D D}_{L P}^{2}-U S \widehat{D D}{ }^{1}{ }_{L P}=\underbrace{\left\lfloor\Delta a_{0}+\Delta a_{1} \overline{\operatorname{Tama\tilde {n}o}}+\Delta a_{2} \overline{\operatorname{Exp}}\right\rfloor}_{\text {Cambio enlos determinantes }}+\underbrace{\left.\mid a_{1} \Delta \overline{\operatorname{Tama\tilde {n}o}}+a_{2} \Delta \overline{\operatorname{Exp}}\right\rfloor}_{\text {Cambio en las característicasde la empresa }}
\end{aligned}
$$

Los resultados de esta descomposición que se realizó usando la ecuación estimada en las primeras dos columnas del Cuadro 3 se muestran en el Cuadro 4. El panel superior indica la descomposición del efecto atribuible a las disparidades entre los niveles de largo plazo para cada período $\left(U S D D_{L P}^{2} \widehat{-}-U S D D^{1} \widehat{L P}\right)$. De acuerdo con nuestra estimación, el nivel de largo plazo de la razón de la deuda en dólares a la total disminuyó 62 puntos porcentuales, de $84 \%$ para el primer período a 22\% para el segundo. Dicha disminución se explica totalmente por el cambio en los determinantes y éste a su vez, por la disminución de la importancia asociada al tamaño de la empresa. Las modificaciones en el resto de los coeficientes y en las características de las empresas se movieron en direcciones opuestas, por lo que eliminaron parte del efecto del cambio en el coeficiente de tamaño.

El panel intermedio del cuadro presenta la disparidad entre los niveles estimados de corto plazo y de largo plazo. La primera fila de este panel presenta el nivel estimado para 1994 y la segunda para 2000. A pesar de que el modelo estimado para el primer período predice un nivel de largo plazo de 84\%, en 1994 fue de tan sólo 38\% debido a que el nivel inicial de la deuda en dólares en 1992 era muy bajo. Algo similar sucede en el segundo período. Ello, toda vez que mientras el modelo predice un nivel estacionario de $22 \%$, el nivel de corto plazo estimado para 2000 fue de $38 \%$ dado el alto nivel observado en 1996. Por tanto, la disparidad entre los niveles de corto y largo plazos durante ambos años implicó un aumento de la deuda en dólares de 62 puntos porcentuales.

De acuerdo con los resultados anteriores, y suponiendo que las variables de tamaño y de exportaciones se mantuvieran constantes, podríamos esperar durante los próximos años una reducción adicional en la relación de deuda en dólares a deuda total. 


\section{Cuadro 4 \\ Factores que determinan el cambio en el endeudamiento en Dlls.}

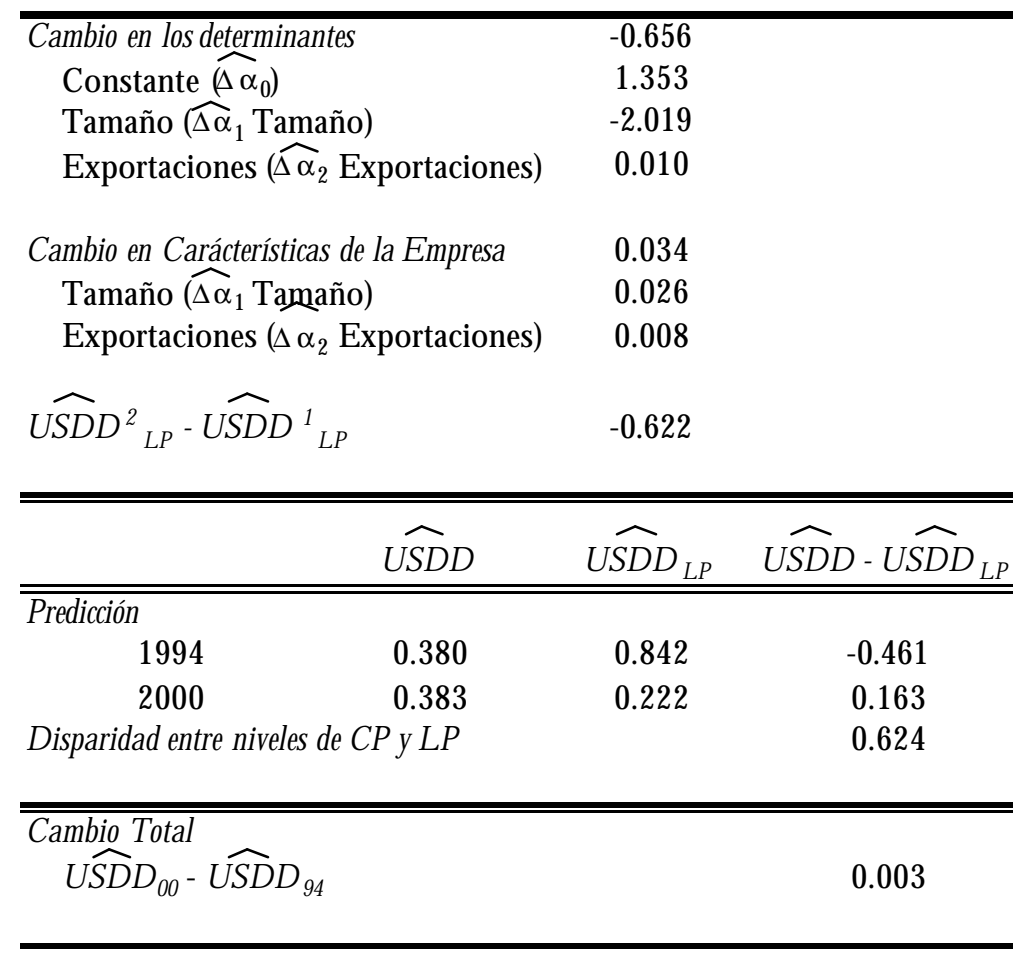

\section{Comentarios Finales}

En este artículo se analiza la hipótesis de que un régimen de tipo de cambio fijo sesga el endeudamiento corporativo hacia préstamos en moneda extranjera. Ello, debido a que se otorga un seguro implícito contra movimientos cambiarios por parte del gobierno. Extendiendo un modelo de información asimétrica desarrollado por Holmstrom y Tirole (1997) se derivó una forma funcional a estimar a fin de encontrar sustento empírico para nuestra teoría. Los resultados indican que las empresas han internalizado en mayor medida el riesgo cambiario desde la adopción del régimen de tipo de cambio flexible. Este argumento se deduce del hecho de que, por un lado, durante el período de régimen de tipo de cambio predeterminado, la relación entre la deuda en dólares y la deuda total estaba determinada principalmente por el tamaño de la empresa. Por otro lado, durante la etapa de libre flotación, las exportaciones se convirtieron en el único determinante significativo de la importancia del endeudamiento en dólares. Incluso, a lo largo de la etapa con tipo de cambio fijo no hubo diferencia alguna entre los ingresos en dólares y en pesos. En 
contraste, durante el período de libre flotación, sólo los ingresos en dólares fueron un determinante importante de la relación entre la deuda en dólares y la deuda total.

Es importante mencionar que aún cuando las empresas están actualmente menos expuestas al riesgo cambiario de lo que estuvieron antes de 1994, aún existe cierto nivel de exposición. Esto se da ya que la mediana de la relación entre las exportaciones y la deuda en dólares está por debajo de $8 \%{ }^{10 /}$. Por tanto, el hecho de que las empresas estén menos expuestas no implica que estén totalmente preparadas para absorber un ajuste significativo del tipo de cambio.

\footnotetext{
${ }^{10 /}$ Antes de la crisis esta relación estaba en su mínimo nivel igual a $2 \%$.
} 


\section{Referencias}

Babatz, Guillermo (1997), “Agency Problems, Ownership Structure, and Voting Structure under Lax Corporate Governance Rules: The Case of Mexico," Tesis Doctoral, Harvard University.

Calvo, G. and C. Reinhart (2000a) "Fear of Floating" NBER - WP 7993. (2000b) "Fixing for your life" Mimeo.

Devereux, M.P., and Schiantarelli, F. (1990), "Investment, Financial Factors, and Cash Flow from UK Panel Data," in G. Hubbard (ed.), Information, Capital Market and Investment, Chicago, IL, University of Chicago Press.

Eichengreen, B. and R. Hausman (1999), "Exchange Rate and Financial Fragility" NBER WP 7418.

Fisher, S.(2001), "Exchange Rate Regimes: Is the Bipolar View Correct?” Mimeo, IMF.

Gelos G. and Werner A. (1999), "Financial Liberalization, Credit Constraints, and Collateral: Investment in the Mexican Manufacturing Sector," IMF Working Paper, No. 9925. Journal of Developments Economics (Febrero 2001).

Harris, J.R., Schiantarelli, F., and Siregar, M.G. (1994), "The Effect of Financial Liberalization on the Capital Structure and Investment Decisions of Indonesian Manufacturing Establishments," The World Bank Economic Review, 8, 17-47.

Holmstrom, B. and J. Tirole (1997), "Financial Intermediation, Loanable Funds and the Real Sector" The Quarterly Journal of Economics, Agosto 1997, Vol. CXII, No. 3 , 663-691.

Mishkin, F.S. (1996), "Understanding Financial Crises: A Developing Country Perspective", NBER Working Paper NO. 5600.

Oliner, S.D., and Rudebusch, G.D., (1992), "Sources of the Financing Hierarchy for Business Investment," The Review of Economics and Statistics, 74(2), 643-54. 